\title{
EVALUATION OF OXIDATIVE STRESS AND ANTIOXIDANT STATUS IN DIABETIC RETINOPATHY CASES
}

Maithri C. M, Aliya Nusrath, Rajeshwari. A.

1. Assistant Professor. Department of Biochemistry, Adichunchanagiri Institute of Medical Sciences, Mandya District, Karnataka, India.

2. Professor \& HOD.. Department of Biochemistry, Adichunchanagiri Institute of Medical Sciences, Mandya District, Karnataka, India.

3. Associate Professor. Department of Biochemistry, Adichunchanagiri Institute of Medical Sciences, Mandya District, Karnataka, India.

\section{CORRESPONDING AUTHOR:}

Dr. Maithri C. M,

Assistant Professor,

Department of Biochemistry,

Adichunchanagiri Institute of Medical Sciences,

B.G.Nagara, Mandya district.

Karnataka state, India - 571448.

E-mail: drmaithri_murali@ymail.com

ABSTRACT: CONTEXT: Diabetes is the most rapidly growing chronic metabolic disorder in the world. In diabetes mellitus, the oxidative stress resulting from enhanced free radical formation and a defect in antioxidant defenses has been implicated in the pathogenesis of diabetes and its complications like neuropathy, retinopathy, nephropathy, etc. AIMS: The objective of the study is to know the oxidative stress by estimating serum malondialdehyde (MDA) levels and antioxidant status by measuring serum vitamin levels, and to know the correlation between MDA and other parameters in cases of diabetic retinopathy. SETTINGS AND DESIGN: Present study involved 100 participants of which 50 were diagnosed as having diabetic retinopathy and 50 were age and sex matched healthy controls. The degree of lipid peroxidation in terms of serum malondialdehyde (MDA) along with antioxidant defenses, vitamin $A$, vitamin $C$ and vitamin E levels were estimated in cases and controls. Fasting blood sugar (FBS), post prandial blood sugar (PPBS) and HbA1c were also estimated in both cases and controls. STATISTICAL ANALYSIS USED: Student t test (two tailed, independent) has been used to find the significance of study parameters on continuous scale between two groups (Inter group analysis) on metric parameters. Pearson correlation between MDA and other parameters was performed to assess the relationship in each group. RESULTS: In diabetic retinopathy patients statistically highly significant increase in levels of FBS, PPBS and HbA1c $(\mathrm{P}<0.001)$ was observed when compared to controls. The levels of serum MDA $(\mathrm{P}<0.001)$ was increased significantly in diabetic retinopathy cases as compared to controls. The levels of serum vitamin $A$, vitamin $C$ and vitamin E were significantly $(\mathrm{P}<0.001)$ reduced in all diabetic retinopathy patients. A positive correlation was observed between MDA and FBS, PPBS, HbA1c. A negative correlation was found between MDA and vitamin A and vitamin C. CONCLUSIONS: The present study concludes that excessive peroxidation damage and antioxidant deficiency occurs in diabetic retinopathy. Further trials regarding the therapeutic measures to control lipid peroxidation and to increase the antioxidant levels are warranted for effective control of diabetic complications.

KEY WORDS: Antioxidants, Diabetic retinopathy, Lipid peroxidation, MDA 
INTRODUCTION: Diabetes mellitus, a lifelong progressive disorder is the result of body's inability to produce insulin or use insulin to its full potential, and is characterized by high circulating glucose. Diabetes is a chronic disease and sustained hyperglycemia attacks both micro vessels and macro vessels throughout the body. It is the leading cause of retinopathy, nephropathy, end-stage renal disease, non traumatic lower extremity amputations and cardiovascular disease. Prevalence of diabetes in adults worldwide was estimated to be $4.0 \%$ in 1995 and is expected to rise to $5.4 \%$ by the year 2025. [1,2] The worldwide prevalence of diabetes mellitus has risen dramatically over the past 2 decades from an estimated 30 million cases in 1985 to 177 million in 2000. Based on the current trends, $>360$ million individuals will have diabetes by the year 2030.[3] In India alone, diabetes is expected to increase from 40.6 million in 2006 to 79.4 million by 2030.[4] Approximately 25\% of patients with diabetes mellitus have been shown to be affected with retinopathy with incidence increasing to $60 \%$ after 5 years and $80 \%$ after 10 to 15 years of affliction. [5] Hyperglycemia and dyslipidemia in diabetes mellitus induces increased lipid peroxidation and reactive oxygen species formation, an important mechanism in the pathogenesis of micro angiopathy. [5] Free radicals or reactive oxygen species (ROS) like superoxide anion radical $\left(\mathrm{O}_{2^{-}}\right)$, hydrogen peroxide $\left(\mathrm{H}_{2} \mathrm{O}_{2}\right)$, alkoxyl (RO), peroxyl (ROO), hydroxyl radical $\left(\mathrm{OH}^{\cdot}\right)$ and hypochlorous acid ( $\mathrm{HOCl}$ ) are highly reactive molecular species with an unpaired electron. The oxidative stress is an imbalance between excess reactive oxygen species formation and impaired removal of reactive oxygen species by antioxidant defense system of the cells like vitamin A, vitamin C, vitamin E, superoxide dismutase, glutathione peroxidase, and catalase. The resulting endogenous oxidative stress causes damage to proteins, lipids and DNA, which is thought to be an important etiological factor in the pathophysiology of complications of diabetes mellitus. [6] Diabetic retinopathy is a progressive disorder and is the most common cause of blindness in people aged 30-60 years. The retina has high content of polyunsaturated fatty acids (PUFA) and has the highest oxygen uptake and glucose oxidation relative to any other tissue. This phenomenon renders retina more susceptible to oxidative stress. Several studies have consistently shown that photochemical retinal injury is attributable to oxidative stress and that the antioxidant vitamins A, E and C protect against this type of injury. [6] Hence the present study was undertaken to evaluate the oxidative status and serum vitamin antioxidants levels in diabetic retinopathy cases.

MATERIAL AND METHODS: Clinically diagnosed 50 cases of diabetic retinopathy cases are included in the study. Age and sex matched 50 healthy individuals are taken as control group. Informed consent was taken and the study was approved by ethical and research committee of the institution. Patients suffering from acute and chronic inflammatory conditions, metabolic conditions like ketoacidosis, cerebrovascular accidents, renal diseases, primary hypertensives, smokers, alcoholics, and psychiatric patients were excluded from the study. None of the subjects were on antioxidant supplementation. $10 \mathrm{ml}$ of fasting blood sample was drawn under aseptic precautions from the study subjects and divided into 3 test tubes. $2 \mathrm{ml}$ of blood with anticoagulant was used for estimation of blood glucose by Glucose Oxidase Method. $2 \mathrm{ml}$ of whole blood was used for estimation of Glycated hemoglobin by Affinity chromatography. $6 \mathrm{ml}$ of plain blood was used for the measurement of

- Serum Malondialdehyde. (Thiobarbuturic acid method)

- Serum Vitamin A. (Spectrophotometric Method)

- Serum Vitamin E. (Baker and Frank method) 
- Serum Vitamin C. (2,4,dinitrophenyl hydrazine method)

Another $2 \mathrm{ml}$ of blood, which was collected with anticoagulant after 2 hrs of meals, was used for estimation of post prandial blood sugar.

STATISTICAL ANALYSIS: Statistical analysis was done using student " $\mathrm{t}$ " test and statistical significance was compared between the cases and the controls. The statistical software namely SAS 9.2, SPSS 15.0, Stata 10.1, MedCalc 9.0.1, Systat 12.0 and R environment ver.2.11.1 were used for the analysis of the data.

RESULTS: Present study included 50 cases of diabetic retinopathy and 50 healthy controls. In both cases and controls fasting blood sugar (FBS), post prandial blood sugar (PPBS), Glycated haemoglobin (HbA1c), Malondialdehyde (MDA), vitamin A, vitamin C and vitamin $\mathrm{E}$ were studied. The age distribution pattern of controls and diabetic retinopathy cases under study ranged from 40 years to 70 years with mean age of $62.0 \pm 9.11$ in controls and $62.12 \pm 7.72$. There was an increase in FBS, PPBS, HbA1c and MDA values in patients as compared to controls which was statistically highly significant $(\mathrm{p}<0.001)$ as shown in the Table/Fig1. There was statistically significant decrease in vitamin $A$, vitamin $C$ and vitamin $E$ values in patients as compared to controls $(\mathrm{p}<0.001)$ as shown in Table/Fig1. Correlation study revealed a small positive correlation between plasma MDA and FBS, PPBS, HbA1c values in cases indicating that as these parameters increases, MDA also increases (Table/Fig 2 and Table/ Fig 3). There was a negative correlation between plasma MDA and vitamin $A$, vitamin $C$ values in cases showing that as MDA increases vitamin $A$ and $C$ value decreases (Table/Fig 2 and Table/ Fig 3).

DISCUSSION: Diabetes mellitus is the chronic degenerative disease of epidemic proportion across the globe. Chronic vascular complications represent the main cause of morbidity and mortality in diabetes mellitus. Free radicals and oxidative stress are found to be responsible for the development of diabetic macroangiopathy and microangiopathy. The impact of microangiopathy in diabetes mellitus includes nephropathy, retinopathy and neuropathy. [6] Diabetic retinopathy is the major cause of blindness in adults. It is a duration dependent disease which develops in stages. In the early stages the diabetic retinopathy is nonproliferative which if left untreated progresses to proliferative diabetic retinopathy. In the present study the mean FBS and PPBS values were $87.74 \pm 12.52$ and $111.16 \pm 10.19$ respectively in controls and $167.70 \pm 60.39$ and $260 \pm 87.99$ in cases respectively, which is statistically highly significant $(\mathrm{P}<0.001)$. Hyperglycemia has been proved to damage the vascular endothelium and the retina by generating ROS. Glycated hemoglobin is a marker for both severity and long term control of disease which reflects the average blood glucose concentration over the preceding 6-8 weeks and is unaffected by diet, exercise, insulin therapy and other drugs. The mean HbA1c values were directly proportional to the risk of developing retinopathy and nephropathy. The normal HbA1c range is $4.2 \%-6.2 \%$. [7] In the present study the mean HbA1c values are $5.60 \pm 0.81$ in controls and $13.34 \pm 1.43$ in cases which is statistically highly significant $(\mathrm{P}<0.001)$. The HbA1c values were higher in cases which correlated well with the clinical diagnosis. The values in the present study are in accordance with several studies which have shown increase in HbA1c levels in diabetes. [8, 9] Development of late diabetic complications is due to both acute and chronic oxidative stress including lipoprotein oxidation, particularly LDL oxidation, peroxidation of PUFA etc. Increased oxidative stress is responsible for both genesis of diabetic retinopathy and progression to advanced stage of proliferative diabetic retinopathy. 
MDA is highly toxic compound formed by lipid peroxidation due to free radical damage. Many studies have shown increase in MDA levels in diabetes mellitus correlating with poor glycemic control. $[5,10]$ In the present study the mean MDA levels were $3.03 \pm 0.84$ in controls and $7.34 \pm 1.71$ in cases which is statistically highly significant $(\mathrm{P}<0.001)$. Madhur M Gupta and Suresh Ghai, in their study showed a significant increase in MDA levels in diabetic retinopathy cases when compared to diabetics without complications and healthy controls. [10] In another study, S Kumari et al, found increase in MDA levels in diabetic retinopathy patients and diabetics without retinopathy patients when compared to controls, however increase in MDA levels in diabetic retinopathy patients was more distinct than in diabetes without retinopathy patients. [5] Vivian Samuel et al, also demonstrated higher levels of MDA in diabetic retinopathy cases as compared to those in diabetics without retinopathy and healthy controls. [11] Gurler B et al and Kesavulu MM have shown increase in lipid peroxide levels with increase duration of disease. [12, 13]

Vitamin A being a component of photoreceptor pigment is needed to maintain the visual process. In the present study the mean vitamin A values are $52.99 \pm 11.64$ in controls and $29.51 \pm 8.71$ in cases which is statistically highly significant $(\mathrm{P}<0.001)$. Present study is in accordance with Zeinab M Osman et al, who found decreased levels of vitamin A and $\beta$ carotene levels in different stages of diabetic retinopathy.[14] Studies have shown that plasma concentration of vitamin A and its carrier proteins (retinal binding protein and transthyretin) are decreased in insulin dependent diabetes mellitus (IDDM).[15] Basu TK et al, in their studies found decreased serum vitamin A levels in IDDM patients and also there was decrease in retinal binding protein levels. [16] From these data it appears that supplementation of vitamin A is beneficial in the treatment and prevention of diabetic retinopathy. The low level of vitamin A in diabetics is attributed to deficiency of intake or defect in transformation of $\beta$ carotene to vitamin $\mathrm{A}$ or defect in metabolic process of vitamin $\mathrm{A}$.

Vitamin $C$ is an important component of antioxidant defense against ROS. In diabetes mellitus there is increased oxidation of ascorbic acid to dehydro ascorbic acid which promotes the oxidative damage. Many studies have shown decreased levels of vitamin $\mathrm{C}$ in diabetes. Vitamin $C$ supplementation has been shown to improve glucose tolerance, decrease lipid profiles, reduces cutaneous capillary fragility, may lower sorbitol levels and there is some evidence that it reduces nonenzymatic glycosylation of proteins. [17] In the present study mean values of vitamin $C$ in cases was $5.17 \pm 2.01$ and in controls it was $12.59 \pm 2.89$ which is statistically highly significant $(\mathrm{P}<0.001)$. S. Kumari et al also showed significant fall in vitamin $\mathrm{C}$ levels in both diabetic with retinopathy and those without retinopathy with more distinct decrease in diabetic retinopathy cases. [5] In another study done by Madhur M gupta and Suresh chari, it was shown that levels of vitamin $\mathrm{C}$ were significantly decreased in diabetics with proliferative retinopathy when compared with the control and diabetic group without complications. [10] Vivian Samuel T et al also showed that level of vitamin C was significantly lower in diabetic group with retinopathy with respect to those of the group without retinopathy. [11]

Vitamin $\mathrm{E}$ is the most important lipophilic membrane antioxidant and prevents lipid peroxidation particularly of PUFA. The decreased level of vitamin E results in damage to internal structures by enhanced free radical production. Vitamin E improves glucose tolerance by improving the activity of insulin within the body. Increase in serum vitamin E levels decreases the likelihood of developing type 2 diabetes as well as reduces the risk of diabetic complications. In the present study the mean vitamin E values were $14.56 \pm 2.61$ in controls and 
$7.05 \pm 2.66$ in cases which is statistically highly significant $(\mathrm{P}<0.001)$. Present study is in accordance with S. Kumari et al, [5] who also showed significant fall in vitamin E levels in diabetic without retinopathy and diabetic with retinopathy however the fall was more distinct in retinopathy cases. Vitamin E supplementation has shown to have a beneficial role in preventing the development of diabetic complications associated with oxidative stress. [18]

Correlation between MDA and other study parameters: Present study revealed a small positive correlation between plasma MDA and both FBS and PPBS in cases ( $r=0.125 ; r=0.111$ ), pointing to contributory role of hyperglycemia towards oxidative stress. This correlation is distorted in cases when compared to controls as there is small negative correlation between MDA and both FBS and PPBS in controls ( $r=-0.178 ; r=-0.191)$. In the present study there was a positive correlation between plasma MDA and HbA1c levels in cases $(r=+0.221)$ indicating that as $\mathrm{HbA1c}$ level increases there is increase in oxidative stress but this correlation is not distorted when cases were compared with controls as controls also showed small positive correlation $(\mathrm{r}=$ +0.140 ) between MDA and HbA1c. In another study, M. Elizabeth et. al showed a positive correlation between HbA1c and glucose in type 1 diabetes mellitus. [9] Correlation study between MDA and vitamin A revealed a moderate negative correlation between MDA and vitamin A levels in both cases and controls $(r=-0.330, r=-0.361$ ) respectively, which was statistically significant. This shows that as MDA level increases vitamin A level decreases. A small negative correlation was also observed between MDA and vitamin $C$ in cases $(r=-0.235)$ which shows that as MDA increase vitamin $C$ value decreases. Present study is in accordance with the study done by S. Kumari et al, who also showed significant negative correlation between plasma MDA and vitamin $\mathrm{C}$, pointing the protective consumption of antioxidant vitamins in the scavenging process. [5] Even though there was a significant decrease in vitamin E levels in cases compared to controls, present correlation study revealed a small positive correlation between MDA and vitamin E $(r=0.179)$ in cases whereas controls showed small negative correlation $(\mathrm{r}=-0.142)$. This could be due to oxidative stress that causes more and more conversion of vitamin $\mathrm{E}$ to vitamin $\mathrm{E}$ radical. This vitamin $\mathrm{E}$ radical interacts with vitamin $\mathrm{C}$ and regenerate vitamin $\mathrm{E}$, hence showing positive correlation between MDA and vitamin $\mathrm{E}$.

Summary and Conclusion: There is increased oxidative stress in diabetes which plays an important role in the pathogenesis of diabetic retinopathy. Present study was undertaken to study the oxidative stress, antioxidant status and to assess whether there is a relationship between oxidative stress and glycemic control in diabetic retinopathy cases. A statistically significant difference was observed in values of FBS, PPBS, HbA1c, MDA, vitamin A, vitamin C and vitamin $\mathrm{E}$ in cases compared to controls. In the present study the positive correlation of MDA with FBS and PPBS pointed out the contributory role of hyperglycemia towards oxidative stress in diabetic retinopathy patients. A small positive correlation was observed between HbA1c and MDA in both cases and controls in the present study which indicates that poor glycemic control increases oxidative stress. The moderate negative correlation between MDA and vitamin A levels that was observed in the present study indicates that as oxidative stress increases, it leads to decreased antioxidant vitamin A levels. Also there was a small negative correlation between plasma MDA and vitamin $\mathrm{C}$ in cases which shows that increase in oxidative stress also leads to decrease in levels of antioxidant vitamin C. Even though the values of vitamin E are significantly less in cases compared to controls, present study showed positive correlation between MDA and vitamin E. However further studies with large sample size is 
required to evaluate the correlation between MDA and vitamin $\mathrm{E}$ to assess the protective role of vitamin $\mathrm{E}$ in preventing oxidative stress.

\section{REFERENCES:}

1. King H, Aubert RE, Herman WH. Global Burden of Diabetes 1995-2025: Prevalence, numerical estimates, and projections. Diabetes Care 1998; 21:1414-31.

2. Ramachandran A, Snehelatha C, Latha E, Vijay V, Viswanathan M. Rising prevalence of NIDDM in an urban population in India. Diabetologia 1997; 40:232-7.

3. Kronenberg HM, Melmed S, Kenneth S, Polonsky, Larsen PR, Williams textbook of endocrinology. 11 $1^{\text {th }}$ ed. Saunders Elsevier publishing Division; 2008

4. Lt Gen Mehta SR, VSM, Col Kashyap AS, Lt. Col Das S. Diabetes Mellitus in India: The Modern Scourge. Medical journal armed forces India 2009; 65: 50-4.

5. Kumari S, Panda S, Mangraj M, Mandal MK, Mahapatra PC. Plasma MDA and antioxidant vitamins in diabetic retinopathy. Indian journal of clinical Biochemistry 2008;23(2):15862.

6. Jakus V. The role of free radicals, oxidative stress and antioxidant system in diabetic vascular diseases. Bratesl Lek Listy 2000; 101(10): 541-51.

7. Trivelli LA, Ranney PH, Lai HT. N Engl J Med 1971; 284.

8. Mc Carter RJ, Hempe JM, Gomez R, Chalew SA. Biological variation in HbA1c predicts risk of retinopathy and nephropathy in type 1 diabetes. Diabetes care 2004; 27:1259-64.

9. Hartnett ME, Rosner BA. Serum markers of oxidative stress and severity of diabetic retinopathy. Diabetes care 2000; 23: 234-40.

10. Gupta MM, Chari S. Lipid peroxidation and antioxidant status in patients with diabetic retinopathy. Indian J of physiol pharmacol 2005; 49(2): 187-92.

11. Samuel VT, Murthy JDS, Dattatreya K, Babu PS, Johncy SS. Impaird antioxidant defence mechanism in diabetic retinopathy. Journal of Clinical and Diagnostic Research [serial online] 2010 December [cited: 2010 December 10]; 4:3430- 6. $w w w . j c d r . n e t / a r t i c l e s / p d f / 1041 / 1462 \_E(C) \_F(J) \_P F(A) \_p . p d f$

12. Gurler B, Vural H, Yilmaz $\mathrm{N}$ et al. Role of oxidative stress in diabetic retinopathy. Eye 2000; 14(5) : 730-7.

13. Kesavulu MM, Giri R, Rao KR et al. Lipid peroxides and antioxidant enzyme levels in type 2 diabetics with microvascular complications. Diabetes and Metab 2000 ; 26(3): 387- 92.

14. Osman ZM, Gomaa AM, Hussein HM, Fardos HS, Soliman, Shobaki AFEL. Association between retinol metabolism and diabetic retinopathy. Polish journal of food and nutrition 2004; 13: 391-6.

15. Basu TK, Basualdo C. Department of agricultural, food and nutritional science, University of Alberta, Edmonton, Canada; Vitamin A homeostasis and diabetes mellitus. 1999 feb; 15(2): 156 www.ncbi.nlm.nih.gov/pubmed/9290094

16. Basu TK, Tze WJ, Leitcher J. Department of food and nutrition, University of Alberta, Edmonton, Canada. Serum Vitamin A and retinol binding protein in patients with insulin dependent diabetes Mellitus. Am J clin Nutr $1989 ; 50(2): 329-31$.

17. Werbach MR. The importance of vitamin $C$ in diabetes mellitus- Nutritional influences on Illness. Townsend letter for doctors and patients Dec 2002. www.highbeam.com/doc/1G1-94538670.html

18. Davis RL, Lavine CL, Arredondo MA, McMahon P, Tenner TE Jr. Differential indicators of diabetes induced oxidative stress in Newzealand white rabbits: Role of dietary vitamin $\mathrm{E}$ 
supplementation. International Journal of Experimental Diabetes Research 2002; 3:18592.

Table/Fig 1: Comparison of variables in two groups

\begin{tabular}{|l|c|c|c|}
\hline Parameters & Controls & Cases & P value \\
\hline *FBS $(\mathrm{mg} / \mathrm{dl})$ & $85.74 \pm 12.52$ & $167.70 \pm 60.39$ & $<0.001^{* *}$ \\
\hline †PPBS $(\mathrm{mg} / \mathrm{dl})$ & $111.16 \pm 10.19$ & $260.86 \pm 87.99$ & $<0.001^{* *}$ \\
\hline †HbA1c\% & $5.60 \pm 0.81$ & $13.34 \pm 1.43$ & $<0.001^{* *}$ \\
\hline §MDA nmol/ml & $3.03 \pm 0.84$ & $7.34 \pm 1.71$ & $<0.001^{* *}$ \\
\hline Vitamin A $\mu \mathrm{g} / 100 \mathrm{ml}$ & $52.99 \pm 11.64$ & $29.51 \pm 8.71$ & $<0.001^{* *}$ \\
\hline Vitamin C mg/L & $12.59 \pm 2.87$ & $5.17 \pm 2.01$ & $<0.001^{* *}$ \\
\hline Vitamin E mg/L & $14.56 \pm 2.61$ & $7.05 \pm 2.66$ & $<0.001^{* *}$ \\
\hline
\end{tabular}

*FBS-Fasting blood glucose †PPBS-Post prandial blood glucose $¥$ HbA1c-Glycated haemoglobin §MDA-Malondialdehyde (** Highly significant)

Table/Fig 2: Correlation of $†$ MDA with other parameters

\begin{tabular}{|l|l|l|l|l|}
\hline \multirow{2}{*}{ Pair } & \multicolumn{2}{l|l}{ Controls } & \multicolumn{2}{l|}{ Case } \\
\cline { 2 - 5 } & $\begin{array}{l}\text { r } \\
\text { value }\end{array}$ & $\begin{array}{l}\text { p } \\
\text { value }\end{array}$ & r value & p value \\
\hline MDA nmol/ml vs łFBS (mg/dl) & -0.178 & 0.216 & 0.125 & 0.386 \\
\hline MDA nmol/ml vs §PPBS (mg/dl) & -0.191 & 0.185 & 0.111 & 0.443 \\
\hline MDA nmol/ml vs $\| \mathrm{HbA1c} \%$ & 0.140 & 0.331 & 0.221 & 0.123 \\
\hline MDA nmol/ml vs Vitamin A $\mu \mathrm{g} / 100 \mathrm{ml}$ & -0.361 & $0.010^{*}$ & -0.330 & $0.019^{*}$ \\
\hline MDA nmol/ml vs Vitamin C mg/L & -0.003 & 0.983 & -0.235 & 0.101 \\
\hline MDA nmol/ml vs Vitamin E mg/L & -0.142 & 0.327 & 0.179 & 0.214 \\
\hline
\end{tabular}

*Significant

†MDA-Malondialdehyde

‡FBS-Fasting blood glucose

§PPBS-Post prandial blood sugar

|| HbA1c-glycated haemoglobin 
Table/Fig 3: Scatter plot for the correlation of *MDA with other parameters

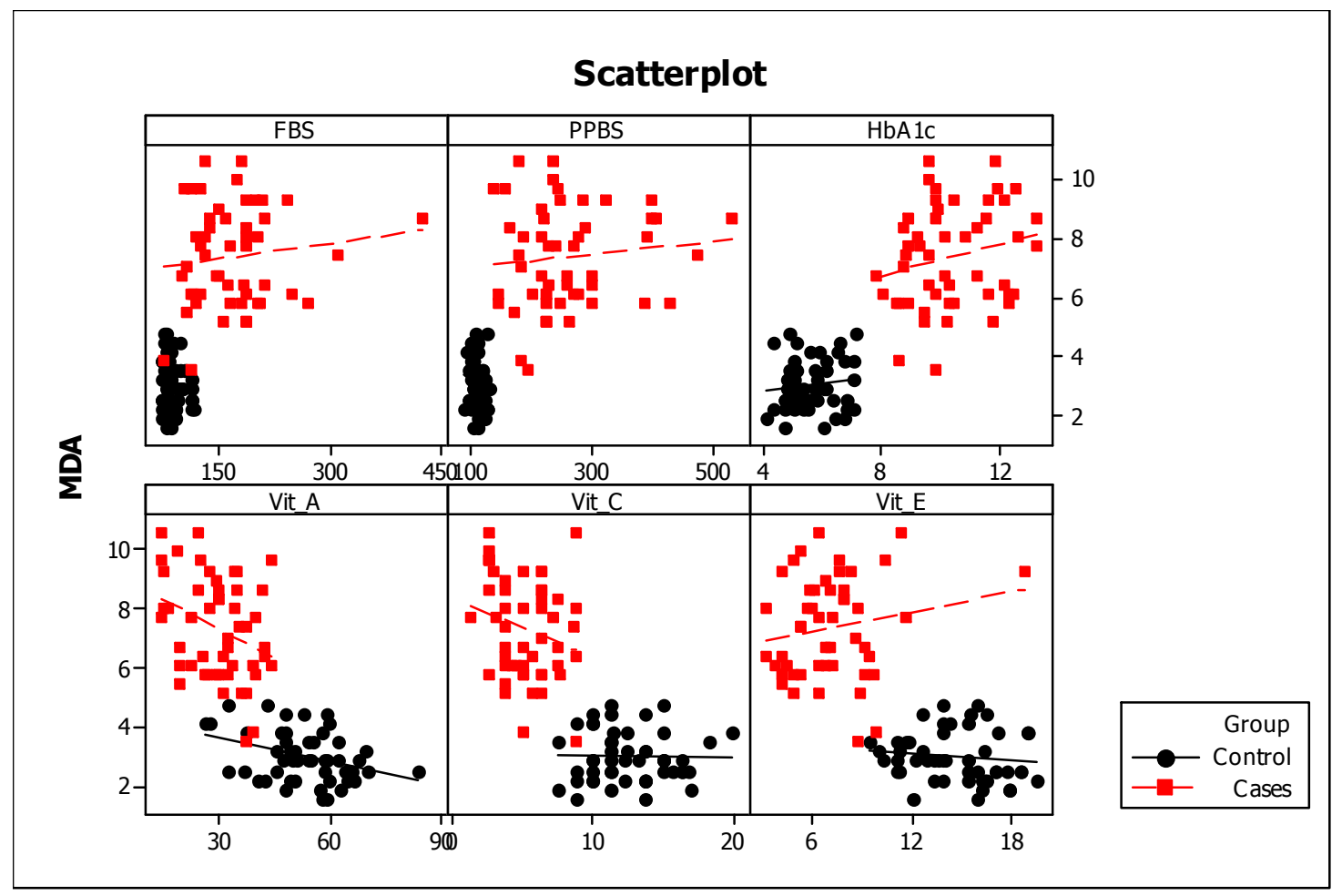

*MDA-Malondialdehyde

\section{Classification of Correlation Co-efficient (r)}

Up to 0.1 Trivial Correlations
0.1-0.3 Small Correlation
0.3-0.5 Moderate Correlation
0.5-0.7 Large Correlation
0.7-0.9 Very Large Correlation
0.9-1.0 Nearly Perfect correlation
1.0 Perfect correlation 